\title{
Mid-Long Term Load Forecasting using Multi-Model Artificial Neural Networks
}

\author{
Reda Mohamed Nezzar ${ }^{1}$, Nadir Farah ${ }^{2}$, Mohamed Tarek Khadir ${ }^{3}$, and Lakhdar Chouireb ${ }^{4}$ \\ ${ }^{1,2,3}$ LabGED University Badji Mokhtar Annaba, Po-Box 12, 23000, Annaba, Algeria. \\ ${ }^{4}$ Societé Nationale d'Electricité et de GAZ (SONELGAZ), Algiers, 16000, Algeria. \\ 1'nezzar@labged.net, ${ }^{2}$ farah@labged.net, ${ }^{3}$ khadir@labged.net, ${ }^{4}$ chouireb.lakhdar@sonelgaz.dz
}

\begin{abstract}
Electrical load is a major input factor in a country's economic development. To support economic growth and meet future demands for electricity, Load forecasting has become a very important task for electrical power management and planning. Several techniques have been employed to accomplish this task. One of those that is mostly used is Artificial Neural Networks (ANNs) method, which have seen the largest number of studies in the field. the load time series data are auto-correlated and are influenced by other variables, such as temperature and population, etc. Furthermore, a large data sample is needed to perform mid-long term forecasts. For the latter purpose and in order to overcome both the lack of data and the high complexity of adding exogenous variables, a multi-model method, based on neural networks, is proposed for the mid-long term load consumption forecasting. The proposed model decomposes, the initial time series into three components using the X12ARIMA algorithm, and performs a forecast for each component using three Nonlinear Auto Regressive (NAR) neural networks, with a Feed Forward neural network combining the outputs of the NARs. The experiments that were conducted showed that the proposed model provided good performance even when using a small sample of available data when compared to benchmark models.
\end{abstract}

Keywords: Mid-long term load forecasting, Feed-forward neural network, Nonlinear Auto Regressive.

\section{Introduction}

Growing countries and modern industries depends heavily on the availability and capacity of electrical power. Which, directly influences a country's economic growth. Therefore, having an accurate medium and long terms electrical load prediction is very important for the electrical industries; it is a crucial input data for all studies of market development of energy production and consumption. Load Forecasting is a step in the planning process of works energy, which determines the investments involved and committed to making irreversible choices on the number of power plants to develop or activate and the number of turbines and other sources of electricity production to install in order to satisfy national and/or regional demand. Effectively, making an accurate forecast a matter of prime advantage [1].

Load forecasting can be performed by a non-linear function approximation, using various variables such as weather, GDP, population growth, etc., along with several other exogenous inputs. In the present study, the focus is on Mid-long term load forecasting, as it directly influences major energy investment decisions, such as the development of new power plans.

Traditionally, statistical models - such as linear regression [2,3] and auto-regressive models [4] - have been widely used in practice for load forecasting, principally because of their simplicity and good performance ratios [5]. However, in recent years, various other models have been proposed. Among these methods, Artificial Intelligence (AI) techniques offer themselves as valuable solutions. The most commonly used AI techniques are ANNs $[6,7,8]$, fuzzy logic $[9,10,11]$, swarm intelligence algorithms $[12,13,14]$, Grey theory $[15,16]$, Wavelet Neural Networks (WNNs) [17,18], Support Vector Machines (SVM) [19], etc.

In this paper, a univariate model was designed, so as to carry out a mid-long term load forecast for the growing Algerian power industry. It is based on different types of neural

Received: May $27^{\text {th }}, 2015$. Accepted: June $10^{\text {th }}, 2016$ 
networks as well as on seasonal decomposition. The above-mentioned combination is found to perform better than other approaches that have already proved their efficiency. The model can deal with little load data and is independent of the exogenous variable, which increases the difficulties in forecasting.

The remainder of this article is organized as follows. A State-of-the-art for used methods in the field of mid-long term forecasting are discussed in the second section. General concepts of Artificial Neural Network (ANN) are presented in the third section. The time series decomposition is described in the fourth section. Section 5 presents the methodology used to develop the proposed X12-NAR-FFNN model in detail. The experiments and results are discussed in Section 6. While, Section 7 concludes the paper.

\section{Related work}

Mid-long term load forecasting is an important foundation of power planning, production and operation. Accurate load forecasting is useful for improving the safety and stability of power network operations; therefore, several models have been developed to provide good forecasts. Some of the most popular approaches are described below.

Zhang and Liu [10] proposed fuzzy rules, obtained using genetic algorithms based on the Takagi-Sugeno Fuzzy Logic System, to forecast loads at mid-long term. The choice of these methods is based on the fact that a Fuzzy logic system provides better forms of rule expression; fuzzy technology provides a reasoning logic that is similar to human reasoning. Genetic algorithms represent an intelligent exploitation of a random search within a defined search space to solve problems. Experiments have verified that this combination, which represents a new and effective model for electrical load forecasting, improves the speed and accuracy of the forecast.

Electrical load forecasting can be affected by various uncertain factors. To determine these, Yue et al. [9] proposed a method based on clustering neural networks exploiting logical operators and fuzzy theory. The new algorithm is constructed and tested using data taken from consecutive years in a district of Guangdong province of China (from 1980 to 2000). Using logical operators and fuzzy theory, the clustering algorithm considers the factors influencing load variations, which improved the speed and accuracy of forecasting.

To overcome the disadvantages (caused by the shortcoming of using a single training set) of using ANNs for mid-long term load forecasting, Yu and Zhang [7] presented a method based on variable structure artificial neural network. This model overcomes some of the intrinsic defects of ANNs, including slow learning speed, difficulties in determining the network structure, and the possible trap of the local minimum. The proposed model has the effect of improving the speed and accuracy by using a stepwise test method to rationally determine network structures and nonlinear wavelets to obtain global optimum fitting.

Fuwei and Wei [20] put forward a new forecasting model using a grey least-squares support vector machine after analyzing the advantages and disadvantages of the grey forecasting method and the Least-Squares Support Vector Machine (LS-SVM). The new model works as follows: first, the accumulation generation for the original sequence is conducted using the Grey forecasting method, then the forecasting model is established to a new sequence with LSSVM; finally, the predicted value is obtained by the inverse accumulated predicted results. The experiment proved that forecasting precision and generalization ability of Grey LS-SVM is better than that of the GM(1,1) model or the LS-SVM model, when used alone; it could offer a considerable improvement in the accuracy of the forecasting.

Ji et al. [17] used a recent machine learning method based on a wavelet neural network (WNN) and a Rough Set. These two methods are combined to establish a rough set-based data pre-processing wavelet network model. The wavelet method is used for load data processing; then a broad application of nerve networks is used for prediction. Their application examples use 13 years of electricity consumption in a Chinese province (data from 1992 to 2005), proving that the proposed model provides good results.

Sun and Zhao [13] proposed a combined model, based on SVM and Continuous Ant- 
Colony Optimization, called MG-CACO, which is used to optimize the parameters of the SVM, reducing the blindness of selection of parameters, and overcoming the shortcomings to determine the parameters by trial and error. Using data from the national statistical yearbook and the Tianjin Statistical Yearbook (of China), they show that the model based on MG-CACO and an SVM is effective in mid-long term load forecasting. The proposed model improves the training speed and increases the prediction accuracy, in comparison with traditional SVM methods.

Lui et al. [21] presented a study of a Grey-based predictive model for long-term forecasting of electrical power demand, and concluded that using the Grey method on its own $[\mathrm{GM}(1,1)]$ is insufficient to handle a volatile electrical system. To overcome the insufficiency, a Grey-RBF neural network forecasting model was proposed by the authors; it improves the Grey prediction and is capable of eliminating the problems of dissipation and overshoots found in general $\operatorname{GM}(1,1)$. Their study contributed to a more robust and reliable model, relative to either the traditional Grey approach or the RBF neural network used alone.

Niu et al. [14] proposed a new model based on the LS-SVM and Particle Swarm Optimization (PSO) for mid-long term forecasting. They applied a mended PSO algorithm to optimize the parameters of the LS-SVM, which avoids opacity when selecting such parameters, and overcomes the phenomena of local optimization. Their experimentation on load data from 1992 to 2007 from Guangxi province in China showed that their method provides better results than any single method or optimal weighted model. The proposed dynamic inertia weight particle swarm optimization algorithm was adopted to improve the parameters of the model, which outperforms standard PSO in terms of speed, and improves the search of optimal global point, which guarantees the efficiency of LS-SVM.

\section{Artificial Neural Network}

The Artificial Neural Network (ANN) is an information processing paradigm inspired by biological nervous systems, which underlie the ways in which the brain processes information. McCulloch and Pitts [22] proposed the first mathematical and computational model of biological neurons in 1943. They were the first to show the formal functions.

The characteristics of ANN information processing make it a powerful computational technique which is able to learn from examples, and then, generalize to others cases that were not presented during training. ANNs are composed of a set of neurons where each neuron is autonomous and undergoes two phases:

- The computation of the weighted sum of the input's in is given by the following equation:

$$
a=\sum_{i} w_{i} \times e_{i}
$$

where $w_{i}$ and $e_{i}$ are respectively the weight and the input of the neuron $i$.

- A transfer function (or activation function) calculating the value of the state of the neuron, based on the previously computed sum.

The strength of ANN's derives from the connections between neurons, which provides the ability to solve non-linear problems based on connections between the different units, several topologies have been proposed in the literature, such as Feed Forward [23], the Recurrent Neural Network [24,1], the Self Organizing Map (SOM) [25], etc. In this study, two types of ANNs are used:

1. The Feed Forward ANN is composed of an input layer where the inputs (represented by $\mathrm{x}$ in the Figure 1) data are fed up in the network, one or more hidden layers, and an output layer. Each layer is composed of more than one neuron and is fully connected to the previous one and each connection has a weight $\left(w_{i}\right)$, as shown in Figure 1. 


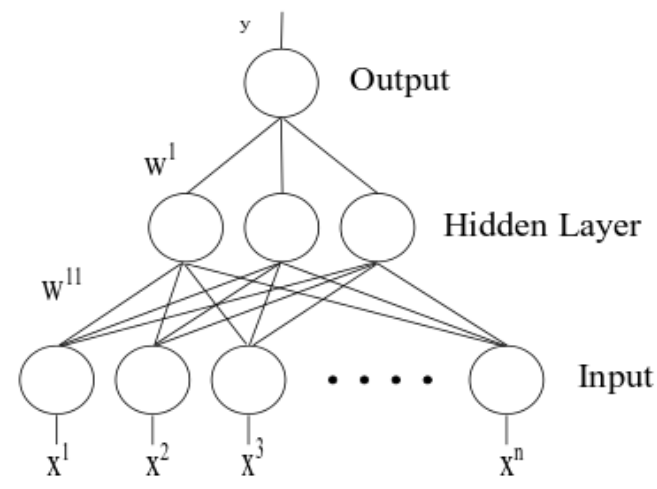

Figure 1. Feed Forward Neural Network.

2. Non-Linear Auto Regressive Neural Network (NAR) (see Figure 2), consisting of a recurrent neural network inspired by the linear Auto Regressive (AR) model, The NAR model is composed by an input, a hidden layer and a one neuron output layer, like the Feed Forward neural network, with the exception that there is a feedback from the output layer to the input layer (shown in Figure 2). The output is given by the following equation:

$$
\hat{y}_{t}=\sum_{i=1}^{d} w_{i} \times y_{t-1}
$$

where $w$ is the parameter to be estimated and $\mathrm{d}$ is the delay. In an AR model, parameters are estimated using Least Mean Squares (LMS). NAR also uses the past values as inputs for the input layer to predict future values, as shown in the following equation:

$$
\hat{y}_{t}=f(y(t-1)+y(t-2)+\ldots+y(t-d))
$$

Instead of using a standard linear regression, the parameters are estimated using a learning algorithm e.g. the Retro propagation algorithm, the Levenberg Marquardt algorithm, or resilient propagation, etc. The added feature of the recurrent model, is the inclusion of past model outputs within the input vector. This ensures multi-step ahead predictions and allows the use of more nonlinear model types such as Nonlinear Box Jenkins.

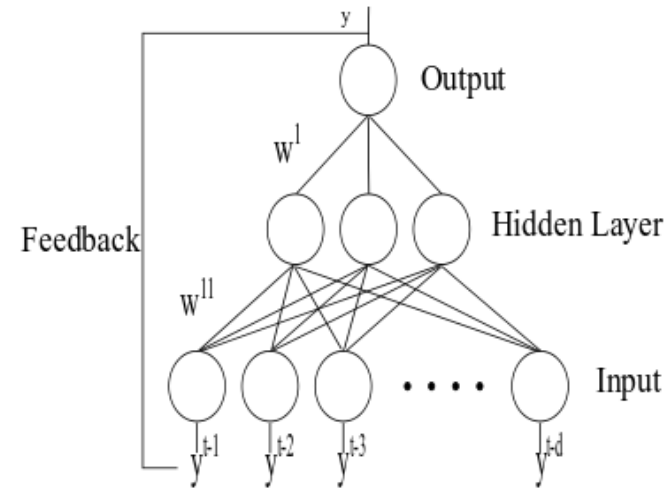

Figure 2. Nonlinear AutoRegressif Neural Network.

\section{Time series decomposition}

A time series may be defined as a sequence of numbers collected at regular intervals over a period of time. Researchers are interested in analyzing the time series to extract meaningful information from data, in order to understand the stochastic process of time series, and variables that influence variations so as to forecast future values. 
The classical decomposition procedure is used in time series analysis to easily extract patterns from the data. Typically, the classical decomposition process takes three components into consideration: the trend cycle $T C_{t}$, the seasonal factor component $S F_{t}$ and the irregular component $I R_{t}$. Those components can be combined into an additive or a multiplicative form as shown below:

$$
\begin{aligned}
& Y_{t}=T C_{t}+S F_{t}+I R_{t} \\
& Y_{t}=T C_{t} \times S F_{t} \times I R_{t}
\end{aligned}
$$

The appropriate form is chosen in accordance with the seasonal variation. It is useful to choose the multiplicative form when seasonal variations increase over time, whereas the additive form should be used when these variations are constant.

\section{Methodology}

Forecasting is necessary in decision-making and operational planning tasks. The difficulty of these tasks varies in accordance with the forecast horizon, and heavily depends on the forecast of exogenous variables affecting future electrical load. The models developed in this direction are called multivariate. Multivariate methods take into account exogenous variables affecting electrical load; forecasting difficulties increase with the number of variables, especially for mid and long-term horizons. For this reason, other models called univariate have been proposed; they exclusively use past load data for forecasting tasks. Univariate methods are not dependent on exogenous variables that are not always available, and should be perfectly predicted in turn, which makes the forecasting task more difficult.

Load series are heavily correlated, and finding external factors that affect the load is typically a difficult task. Therefore, the main objective of the proposed study, is to perform an accurate and reliable load forecasting with minimal modifications to the original data to design a model which can fulfil a forecast with minimal sample data. This goal is achieved using a combination of seasonal decomposition and neural networks called X12- NAR-FNN; the later use monthly data to forecast the Algerian load in the mid-long terms.

The proposed model is a process of three steps, where the original load goes through each step and the load is changed in consequence, and the output of a step is the input of the next one. Three methods are used in the X12-NAR-FFNN model: X12-ARIMA decomposition, NAR and a Feed Forward neural network. These methods are organized as follows (see Figure 3):

- A decomposition of the original data into three components: SF, IR and TC via the multiplicative model of the X12-ARIMA algorithm.

- A monthly forecast of SF, TC and IR components is achieved via three NAR neural networks; the final 12 months of each component are used as inputs for each neural network to capture the yearly pattern. The end of this step results in monthly forecasts of SF, TC, and IR for a complete year.

- Finally, the Feed Forward ANN combines the outputs of the three NAR networks for a 12 months ahead load forecast.

All of the proposed models in this study were created using data ranging from 2000 to 2010, which provided us with 132 monthly observations, which are used for the training and validation phases, whereas the rest of the observations (from the years 2011 and 2012) will be used for the testing phase. 


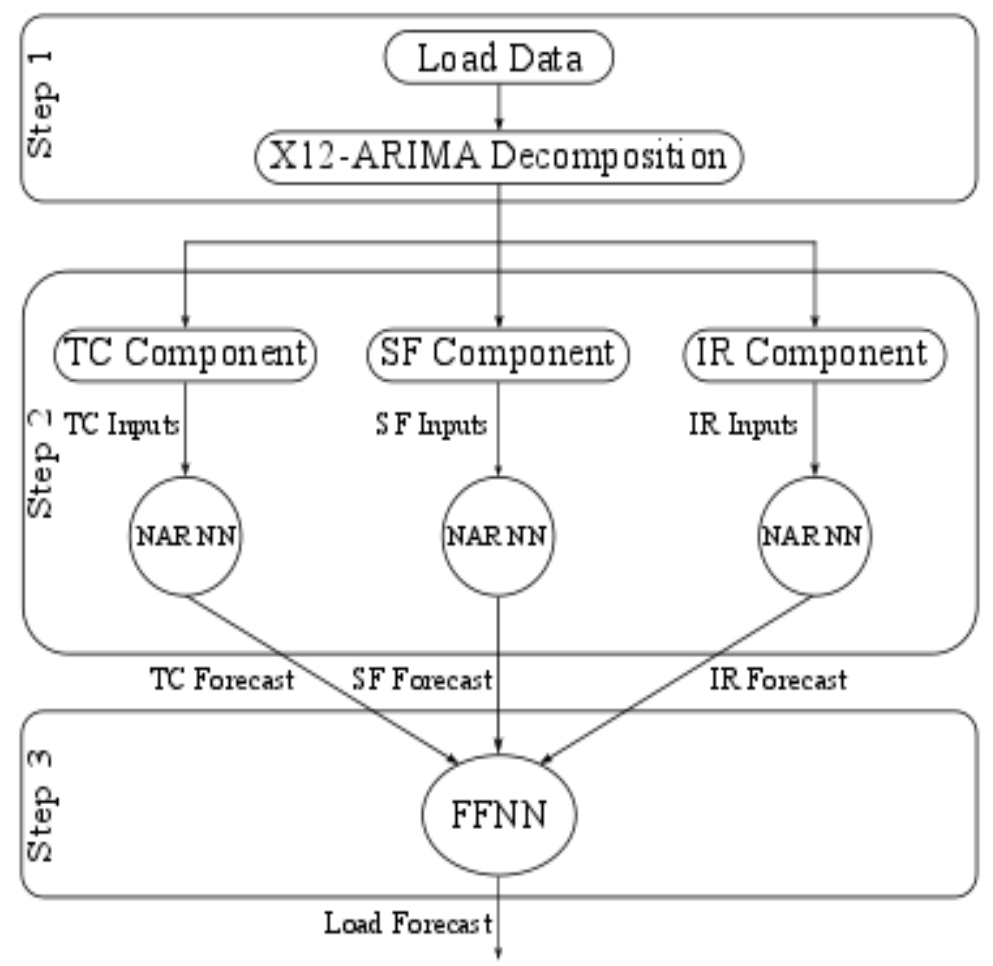

Figure 3. The X12-NAR-FFNN Model

\section{Experimentation and Results}

A. Used dataset and preprocessing

The dataset was provided by the Algerian power production company (Sonelgaz) and represents the daily load from January 1, 2000 to December 31, 2012, sampled every 15 minutes. To perform a good mid-long term forecast, it is preferable to use monthly, instead of daily, data. Therefore, it is necessary to perform a re-sampling, taking the peak of each month of the original data. As a result, we obtain a total of 156 observations, with 12 values for each year, and a total of 156 values for the 13 years used.

Classical decomposition is often used, but suffers from several inconveniences; among these, there is no trend estimation for the first and last observations. The decomposition reduces the original data, which, then, became inappropriate when there are few observations. It is assumed that the seasonal component repeats itself from year to year. For many series, this is not a reasonable assumption. Several methods have been using classical decomposition as a base to overcome the drawbacks of the original approach; among them are X12-ARIMA, RMA, etc.

The X12-ARIMA model was developed by the United States Bureau of the Census (1998) [26] and is considered in this work as a method for monthly load data decomposition, enabling us to obtain a trend estimation for all observations and a seasonal component that can vary slowly over time, with a relative robustness to unusual observations. This method is based on a model called regression-arima, and is used to improve forecasting and seasonal adjustment. Then, the X11 model [27] handles the outputs of the regression-ARIMA model to extract TC, IR and SF from the time series.

\section{B. Performance measures}

A comparison of the proposed model X12-NAR-FFNN is performed in this study with other models, such as NAR, and Least Squares Support Vector Regression LSSVR, multi- 
models like SD(M)-LSSVR-LSSVR [28], SD(M)-LSSVR and X12-NAR. The comparison was based on different types of criteria among them: Mean Absolute Percentage Error (MAPE), a measure of accuracy for constructing fitted time series values. MAPE is used to calculate the prediction accuracy of each model and is defined by the formula in Equation 6:

$$
M A P E=\frac{1}{N} \times \sum_{i=1}^{N}\left|\frac{x_{t}-\hat{x}_{t}}{x_{t}}\right|
$$

where $x_{t}$ is the actual value and $\hat{x}_{t}$ is the forecast value. The difference between $x_{t}$ and $\hat{x}_{t}$ is divided by the actual value $x_{t}$ again. The absolute value in this calculation is summed for every fitted or forecasted point in time and divided again by the number of fitted points $n$. This is multiplied by 100 , given a percentage error. To obtain a comprehensive and valuable study of the predictive effect of all the tested models, other error indexes were added, consisting in: Root-Mean-Square Error (RMSE) [29] and Mean Absolute Error (MAE) [30].

In addition to the forecast accuracy, another comparison of the models on their ability to predict movement direction is performed, using Directional statistic test $D_{\text {stat }}$ and is formulated in Equation (7):

$$
D_{\text {stat }}=\frac{1}{N} \sum_{t=1}^{N} a_{t} \times 100 \%
$$

where $a_{t}=1$ when the product $\left(x_{t+1}-x_{t}\right) \times\left(\hat{x}_{t+1}-x_{t}\right)>1$, otherwise $a_{t}=0$.

\section{Experimentation and Discussion}

In order to validate the results and the reliability of the X12-NAR-FFNN models, we perform a comparison with the proposed model by Wang et al. [28], which forecast hydropower consumption in China. The benchmark model is based on seasonal decomposition (SD) with the X12-ARIMA algorithm and Least Square Support Vector Regression (LSSVR) method. The model, called SD(M)-LSSVR-LSSVR, first decomposes the hydropower series into three components, which are, in turn, predicted using three LSSVR models and a fourth LSSVR, combining predictions of the three components to get the final hydro-power consumption forecast. The authors proved the efficiency of the X12-ARIMA method by comparing it with other decomposition methods, such as the Ratio to Moving Average (RMA) and the Empirical Mode Decomposition (EMD). Also, the authors found that the model's performance decreases when the forecasting horizon is large.

Therefore, to perform a comparison of the X12-NAR-FFNN proposed model, a selection of model based on two approaches were used:

- Models based on neural networks; a simple regressive model (NAR) and an X12-NAR model, which breaks down the original time series into three components (i.e. TC, SF, and IR) (see Section 5) via the X12-ARIMA algorithm. Then, each component is predicted using the NAR model. Finally, to obtain the final load, a multiplication of the predicted components is performed: all NARs have 12 input neurons to catch the yearly pattern of the of each seasonal components and 12 neurons in the hidden layer and one output neuron; the sigmoid activation function and the Resilient Propagation Algorithm were used for all neural models.

- Models based on LSSVR; a simple LSSVR model and an X12-LSSVR model, which follows the same approach compared to the one used for the X12-NAR model. However, instead of neural networks, LSSVR was used, in addition to the benchmark model, called SD(M)-LSSVR-LSSVR. A Gaussian RBF kernel is used for all LSSVR models presented in this study.

A comparison between the neural models and LSSVR models is conducted. The later proved their efficiency and their ability to learn and forecast mid-long term horizons, with a small sample data [28]. After re-sampling the Algerian daily load into months, NAR and LSSVR models were trained, using data from January 2000 to December 2010. Evaluation 
methods, with the performance measures described above, have been carried out on several horizons; 3, 6, 12 and 24 months.

The experimentation was conducted in three parts. In each part, the Neural Network and LSSVR learning paradigm are compared. The first part evaluates the NAR and LSSVR model and using MAPE, RMSE and MAE error, the latter gives the respective errors $4.44 \%, 557.11$, 377.05 and is proven to be more efficient than the NAR model, which reaches respective errors of $5.40 \%, 626.13$ and 456.13 for a 24 -month horizon (see Table 1). The LSSVR model is more efficient than the NAR in the mid-long term because it retains the principle of SRM and has good generalization capabilities. Although, both have the same $D_{\text {stat }}$, with a percentage of $78.28 \%$ (see Figure 6).

The second part of the experiment is based on the load decomposition into Trend Cycle (TC), Seasonal Factor (SF) and Irregular Components (IR) (as shown in Figure. 4), using the multiplicative model of the X12-ARIMA algorithm and its added value when compared with a simple model. Subsequently, three NAR and LSSVR models were used to predict TC, SF and IR called respectively X12-NAR and SD(M)-LSSVR. The outputs of the later are the result of the product of the three components obtained from NAR and LSSVR models, with $2.49 \%$, 250.22, 199.86 for respectively MAPE, RMSE and MAE errors, respectively, and a Dstat of $86.9 \%$ for the 24-month horizon. The X12-NAR is more accurate than SD(M)-LSSVR, which reaches an error of $3.08 \%, 353.40$ and 254.46 for the same criteria, and a $D_{\text {stat }}$ of $82.6 \%$.

The decomposition of the load allows the neural networks based model, which are known to be data-greedy, to be more efficient with a small set of learning data and results in nearly $3 \%$ more MAPE errors than the simple NAR models (see Figure. 5). The X12-NAR outperform the SD(M)-LSSVR for all horizons, as shown in Table 1 and is more efficient for predicting data movement direction.

Table 1. Performances measures of models on 3, 6, 12 and 24 months horizon.

\begin{tabular}{|c|c|c|c|c|c|c|c|}
\hline & & 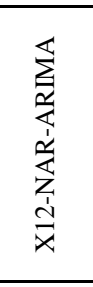 & 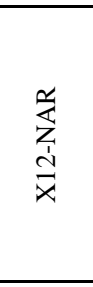 & 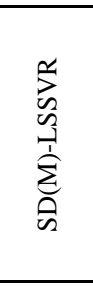 & 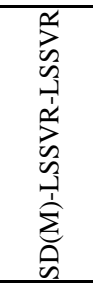 & 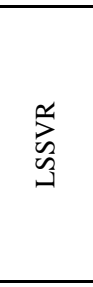 & $\underset{z}{\frac{2}{z}}$ \\
\hline \multirow{3}{*}{$\sum_{m}$} & MAPE & 1.25 & 0.77 & 1.27 & 1.28 & 1.92 & 2.77 \\
\hline & RMSE & 115.07 & 63.24 & 116.52 & 117.17 & 146.55 & 235.18 \\
\hline & MAE & 95.30 & 59.10 & 98.03 & 98.43 & 146.49 & 209.49 \\
\hline \multirow{3}{*}{$\sum_{0}$} & MAPE & 2.12 & 1.84 & 2.43 & 2.40 & 2.91 & 2.26 \\
\hline & RMSE & 184.03 & 148.53 & 205.73 & 205.60 & 258.73 & 188.30 \\
\hline & MAE & 151.85 & 127.89 & 170.65 & 169.16 & 210.56 & 164.45 \\
\hline \multirow{3}{*}{$\underset{工}{\sum}$} & MAPE & 2.00 & 2.11 & 2.45 & 2.46 & 3.60 & 3.28 \\
\hline & RMSE & 220.39 & 198.46 & 220.92 & 222.33 & 328.22 & 322.32 \\
\hline & MAE & 150.06 & 157.76 & 181.47 & 182.66 & 274.93 & 254.92 \\
\hline \multirow{3}{*}{$\underset{\sim}{\sum_{\sim}}$} & MAPE & 2.35 & 2.49 & 3.08 & 3.16 & 4.44 & 5.40 \\
\hline & RMSE & 270.95 & 250.22 & 353.40 & 358.83 & 557.11 & 626.43 \\
\hline & MAE & 189.65 & 199.86 & 254.46 & 260.71 & 377.05 & 456.13 \\
\hline
\end{tabular}

The results show that the X12-ARIMA decomposition increased the accuracy of the forecast and uses a small set of learning data. Adding a LSSVR model to combine the three components does not improve the performance of the prediction errors, and $D_{\text {stat }}$ is almost equal to that of SD(M)-LSSVR. In contrast to SD(M)-LSSVR-LSSVR, the X12-NAR- FFNN 
model improves the prediction error when compared to X12-NAR to obtain a MAPE, RMSE, and MAE value of respectively $2.35 \%, 270.95,189.65$ and $D_{\text {stat }}$ of $91.30 \%$, making it the best performing model for all horizons. Figure 5 and 6 respectively summarize the MAPE criteria error cited above and $D_{\text {stat }}$ of all models for a 24-month horizon, and shows clearly the X12NAR-FFNN models outperforms in term of error accuracy and directional movement of the forecast than the other models used in this study.
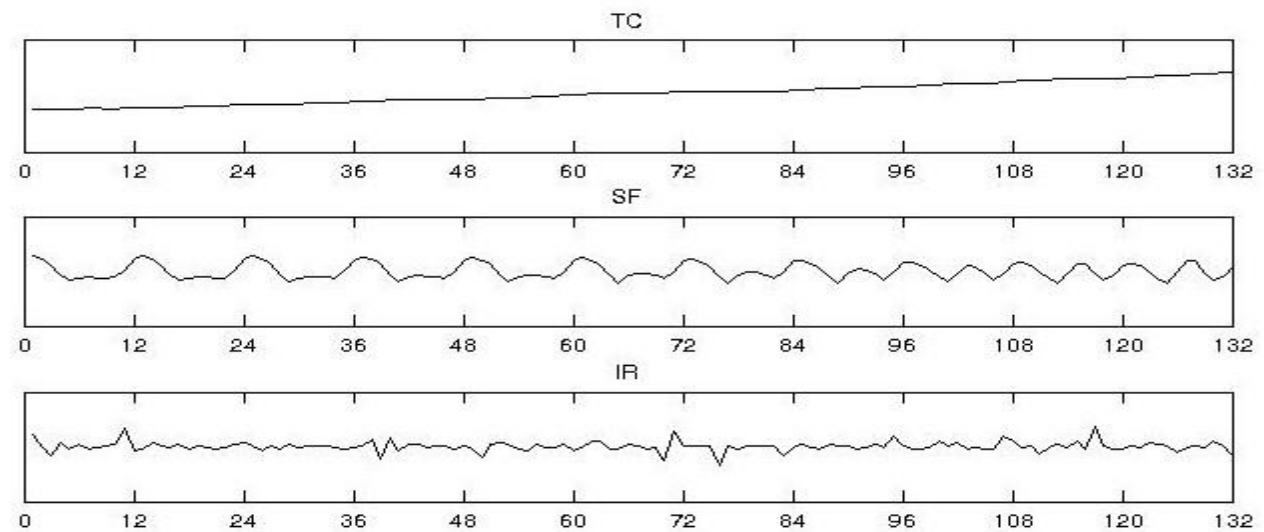

Figure 4. Example of Trend-Cycle, Seasonal Factor and Irregular component.

The load forecast from January 2011 to December 2012, given by X12-NAR- FFNN, is shown in Figure. 7. The model is shown to be valid when compared to a real load, including the increase observed by the end of 2012 due to the growth of both the housing sector and Algerian industries. Neural networks have proven their efficiency in a number of scenarios in the fields of forecasting electrical loads, but like any mathematical model they have advantages and disadvantages. For this reason, the tendency is moving towards multi-models in order to combine different model's best features. The proposed X12-NAR-FFNN model is capable of learning from a small sample of data and can forecast for up to 24 months. This is model is free from exogenous variables. Furthermore, the decomposition process embedded in the model makes it more robust to incorrect values in the time series.

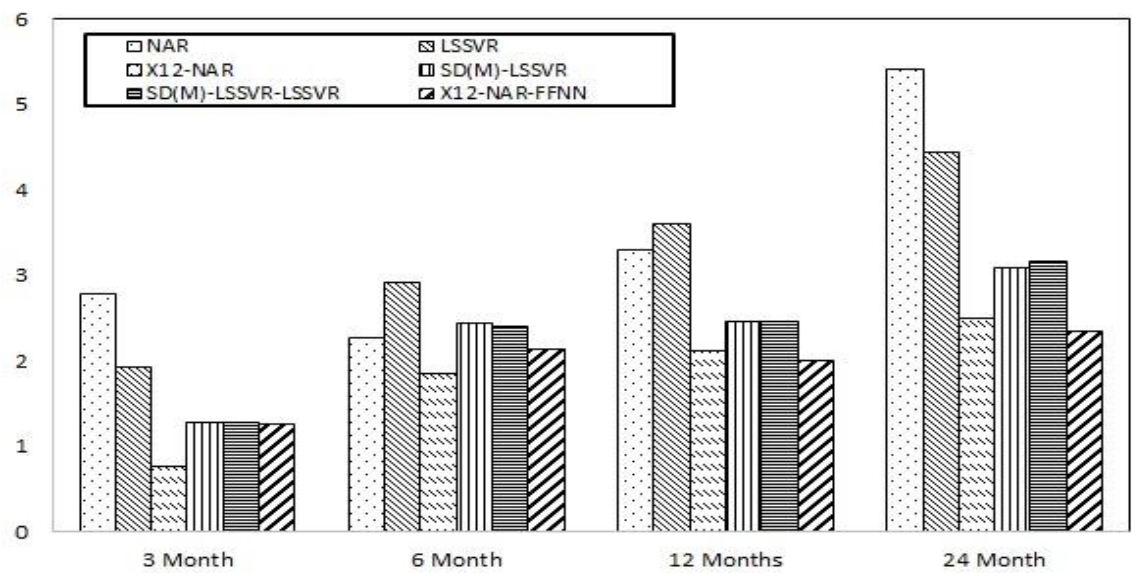

Figure 5. The MAPE error of the models.

One important parameter for the energy industry is to predict the annual peak load so as to meet future demand. The mid-long term forecast allows energy suppliers to predict whether the current facilities can satisfy future demand; if not, it becomes necessary to plan the construction of new power plants. In this case the X12-NAR-FFNN reaches the peak of August 
of 2011 and 2012, in addition to the seasonal and trends, which are well reproduced by the proposed model (Figure. 7). X12-NAR-FFNN also has the best MAPE, RMSE, MAE and $D_{\text {stat }}$ score making it the best model in term of prediction accuracy among models that have already proven their efficiency, such as the SD(M)-LSSVR-LSSVR model.

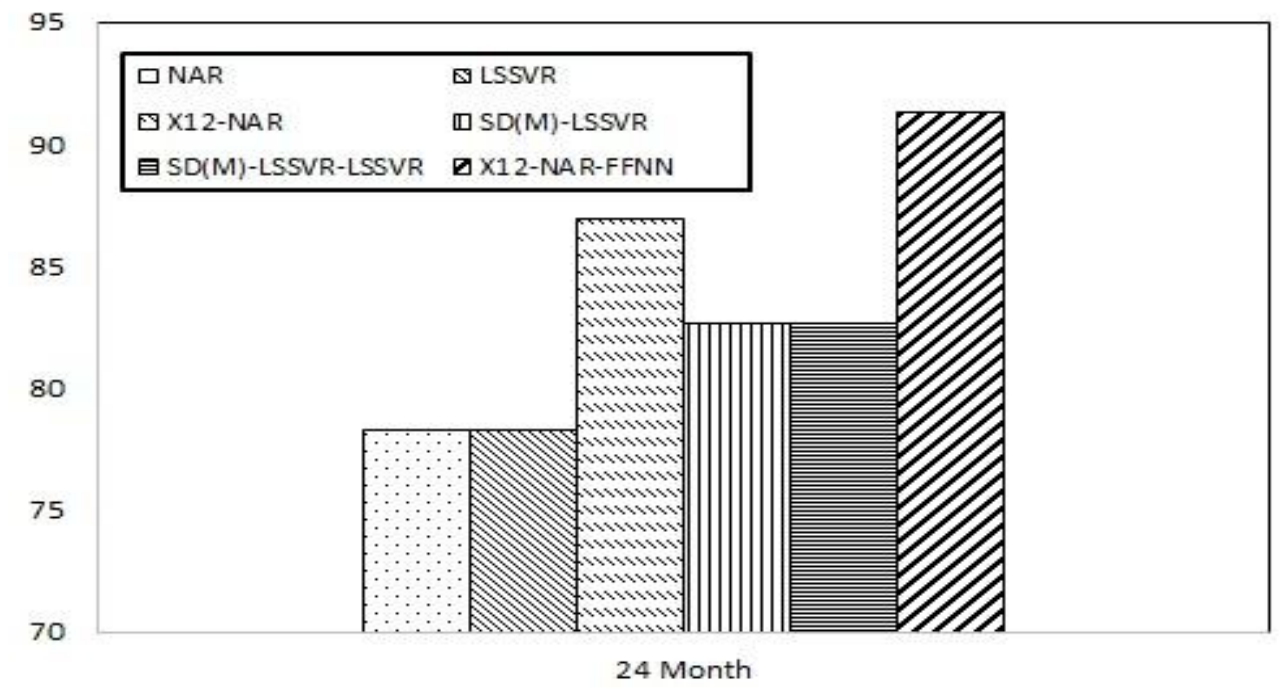

Figure 6. Directional statistic of all the model for 24 months horizon.

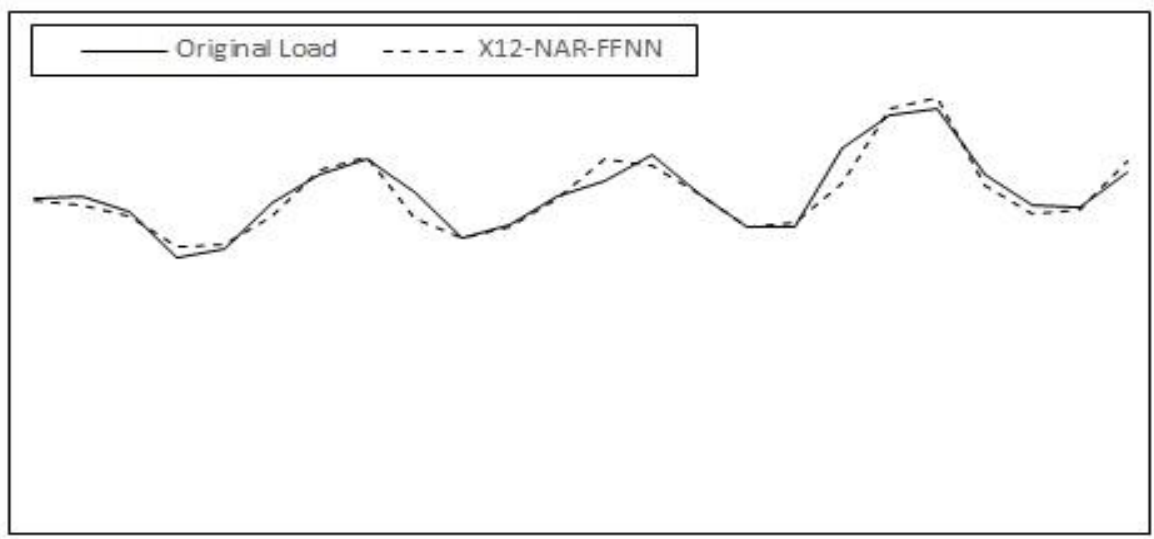

$\begin{array}{llllllllllllllllllllllll}1 & 2 & 3 & 4 & 5 & 6 & 7 & 8 & 9 & 10 & 11 & 12 & 13 & 14 & 15 & 16 & 17 & 18 & 19 & 20 & 21 & 22 & 23 & 24\end{array}$

Figure 7. The load forecast from January 2011 to December 2012 of the X12-NAR-FFNN.

\section{Conclusion}

Load is heavily dependent on exogenous variables and model design. For medium and long term, it implies incorporating these variables, which is often form a complicated task. Hence, the interest in univariate models, which require only past load values. ANNs have been widely used for load forecasting; however, they have the disadvantage of being greedy in terms of training data.

In this study, a univariate multi-model based on neural networks has been proposed for the forecasting of the Algerian electrical load for the mid-long terms using a small training data sample (January 2000 to December 2010). On the one hand, the X12-NAR-FFNN model outperforms its competitors for a 24-month forecast (from January 2011 to December 2012), and it can be deduced, from the results obtained, that seasonal decomposition considerably increases the forecast performances. On the other hand, the combination of NAR neural 
networks and FFNN provides conclusive results for the mid-long term, when compared to other models with proven efficiency in the literature.

\section{Acknowledgements}

The authors would like to express their sincere gratitude to SONALGAZ for providing valuable data and its employees for their reliability, confidence and cooperation.

\section{References}

[1]. J. Connor, R. Martin and L. Atlas, "Recurrent neural networks and robust time series prediction", IEEE Transactions on Neural Networks, Vol. 5, No. 2, pp. 240-254, Mar. 1994.

[2]. N. Abu-Shikhah, F. Elkarmi and O. M. Aloquili, "Medium-term electric load forecasting using multivariable linear and non-linear regression", Smart Grid and Renewable Energy, Vol. 2, No. 2, pp. 126-135, May. 2011.

[3]. D. Niu, Y. Lu and Y. Wei, "Research on mid-long term power load forecasting models of Anhui province”, International Journal of Advancements in Computing Technology, Vol. 5, No. 5, pp. 535-542, Mar. 2013.

[4]. H. Al-Hamadi and S. Soliman, "Long-term/mid-term electric load forecasting based on short-term correlation and annual growth", Electric power systems research, Vol. 74, No. 3, pp. 353-361, Jun. 2005.

[5]. R. Weron, "Modeling and forecasting electricity loads and prices: A statistical approach", West Sussex, England: John Wiley \& Sons, 2007.

[6]. M. Ghiassi, D. K. Zimbra and H. Saidane, "Medium term system load forecasting with a dynamic artificial neural network model", Electric power systems research, Vol. 76, No. 5, pp. 302-316, Mar. 2006.

[7]. H. Yu and Q. Zhang, "Application of variable structure artificial neural network for midlong term load forecasting", The 2nd IEEE International Conference on Information Management and Engineering, Chengdu, pp. 450-453, Apr. 2010.

[8]. C. Xia, J. Wang and K. McMenemy, "Short, medium and long term load forecasting model and virtual load forecaster based on radial basis function neural networks", International Journal of Electrical Power \& Energy Systems, Vol. 32, No. 7, pp. 743-750, Sep. 2010.

[9]. L. Yue, Y. Zhang, H. Xie and Q. Zhong, "The fuzzy logic clustering neural network approach for middle and long term load forecasting", IEEE International Conference on Grey Systems and Intelligent Services, Nanjing, pp. 963-967, Nov. 2007.

[10]. Q. Zhang and T. Liu, "Research on the mid-long term electric load forecasting based on fuzzy rules", 2nd IEEE International Conference on Information Management and Engineering, Chengdu, pp. 461-463, Apr. 2010.

[11]. W. Biao, F. Gang, H. Jing and X. Xian-Yong, "Electric power load forecasting based on fuzzy optimal theory", Second International Conference on Intelligent System Design and Engineering Application, Sanya, pp. 821-824, Jan. 2012.

[12]. G. Pan and A. Ouyang, "Medium and long term power load forecasting using CPSO-GM model", Journal of Networks, Vol. 9, No. 8, pp. 2121-2128, Aug. 2014.

[13]. W. Sun and W. Zhao, "Mid-long term power load forecasting based on MG-CACO and SVM method", 2nd International Conference on Future Computer and Communication, Wuhan, pp.V1-118-V1-121, May. 2010.

[14]. D. Niu, H. Lv and Y. Zhang, "Combination forecasting model for mid-long term load based on least squares support vector machines and a mended particle swarm optimization algorithm", International Joint Conference on Bioinformatics, Systems Biology and Intelligent Computing, Shanghai, pp. 525-528, Aug. 2009.

[15]. H. Zhang, Z. G. Lei, Y. Cheng and Y. M. Wang, "Medium and long-term load forecasting using grey theory based on rough sets", Applied Mechanics and Materials, Vol. 672, pp. 1405-1408, Oct. 2014. 
[16]. X. S. He, L. Yang and X.N. Yu, "Research of combined optimum grey model to mid and long term electric load forecasting", Advanced Materials Research, Vol. 986, pp. 13791382, Jul. 2014.

[17]. Z. Ji, P. Zhang and Z. Zhao, "Application of wavelet neutral network and rough set theory to forecast mid-long-term electric power load", First International Workshop on Education Technology and Computer Science, Wuhan, pp. 1104-1108, Mar. 2009.

[18]. Q. Zhang and T. Liu, "A fuzzy rules and wavelet neural network method for mid-long term electric load forecasting", Second International Conference on Computer and Network Technology, Bangkok, pp. 442-446, Apr. 2010.

[19]. W. C. Hong, "Electric load forecasting by support vector model", Applied Mathematical Modelling, Vol. 33, No. 5, pp. 2444-2454, May. 2009.

[20]. Z. Fuwei and C. Wei, "Application of grey LS-SVM in mid and long term power load forecasting", International Conference on Management and Service Science, Wuhan, pp. 1-4, Sept. 2009.

[21]. H. Liu, L. Cai and X. Wu, "Grey-RBF neural network prediction model for city electricity demand forecasting", 4th International Conference on Wireless Communications, Networking and Mobile Computing, Dalian, pp. 1-5, October 2008.

[22]. W. S. McCulloch and W. Pitts, "A logical calculus of the ideas immanent in nervous activity”, The bulletin of mathematical biophysics, Vol. 5, No. 4, pp. 115-133, Dec. 1943.

[23].Z. Tang and P. A. Fishwick, "Feedforward neural nets as models for time series forecasting”, ORSA Journal on Computing, Vol. 5, No. 4, pp. 374-385, Nov. 1993.

[24]. G. Dorffner, "Neural networks for time series processing", Neural Network World, Vol. 6, No. 4, pp. 447-468, 1996.

[25]. Kohonen T, "The self-organizing map", Neurocomputing, Vol. 21, No. 1, pp. 1-6, Nov. 1998.

[26]. D. F. Findley, B. C. Monsell, W. R. Bell, M. C. Otto and B. C. Chen, "New capabilities and methods of the X-12-ARIMA seasonal-adjustment program", Journal of Business \& Economic Statistics, Vol. 16, No. 2, pp. 127-152, Apr. 1998.

[27]. E. B. Dagum, "Modelling, Forecasting and seasonally adjusting economic time series with the X-11 ARIMA method", Journal of the Royal Statistical Society, Vol. 27, pp. 203$216,1978$.

[28]. S. Wang, L. Yu, L. Tang and S. Wang, "A novel seasonal decomposition based least squares support vector regression ensemble learning approach for hydropower consumption forecasting in china", Energy, Vol. 36, No. 11, pp. 6542- 6554, Nov. 2011.

[29]. Chen and Toly, "A collaborative fuzzy-neural approach for long-term load forecasting in Taiwan”, Computers and Industrial Engineering, Vol. 63, No. 3, pp. 663-670, 2012.

[30]. J. Hu, J. Wang, and G. Zeng, "A hybrid forecasting approach applied to wind speed time series", Renewable Energy, Vol. 60, pp. 185-194, Dec. 2013. 


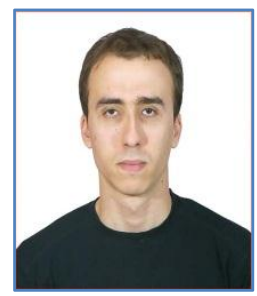

Reda Mohamed Nezzar was born on November 10, 1986 in Annaba, Algeria. After succeeding his baccalaureate in 2004, he graduated from university of Badji Mokhtar Annaba, Algeria with a bachelor degree in Computer Science in 2008, he undertook a Science and Technologies of Information and Communication (STIC) Master degree in 2010 where he worked on semantic web and mashups. Nezzar Med Reda started a Ph.D in September 2010 affiliated to the LABGED laboratory, with his thesis director Farah Nadir they are working on load forecasting of their native country. His research interests are Artificial Intelligence, Artificial Neural Network, load forecasting.

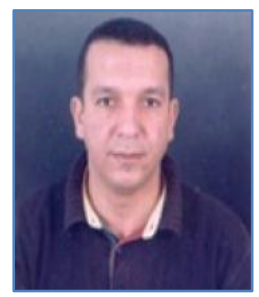

Nadir Farah was born on May 26, 1966 in Annaba, Algerian. After succeeding his bacalaureat in 1984, he graduated from university of Badji Mokhtar Annaba, Algeria, with a state Engineering degree in Computer Science, in 1989, He undertook an M.Sc at Badji Mokhtar University with first class honors in 1994. Farah Nadir received a Ph.D. degree from the same University in 2001. He then continued with this institution as a teacher, senior lecturer and researcher in LABGED laboratory, this last is focusing on the management of electronic document. He succeeded in obtaining the HDR (Habilitation to Direct Research) in January 2005, conferring him the title of Senior lecturer. Since 2010 he is the director of LABGED laboratory and in January 2010 he was promoted full professor. His research interests are pattern recognition, character/word recognition, signal processing and bioinformatics.

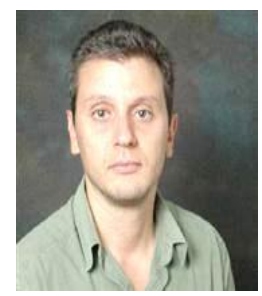

Mohamed Tarek Khadir was born on the 5th of July 1972 in Annaba Algeria. After succeeding in the baccalaureate, majoring in Maths and Technology in 1989, he graduated from the University of Badji Mokhtar Annaba, Algeria, with a state Engineering degree in Electronics Majoring in Control, in 1995. After two years' work in the computer industry, he undertook an M.Eng. at Dublin City University, Ireland Graduating with First class honors in 1998. Tarek Khadir received a Ph.D. degree from National University of Ireland, Maynooth, Ireland in 2002. Then, he continued with this institution as a post-doctoral researcher until September 2003 when he joined the department of computer science at university Badji Mokhtar Annaba, Algeria, as a senior lecturer. He succeeded in obtaining the HDR (Habilitation to Direct Research) in January 2005, conferring him the title of Senior lecturer. In January 2010 he was promoted full professor. His main interest are Artificial Neural Networks, Artificial Intelligence and Industrial Engineering.

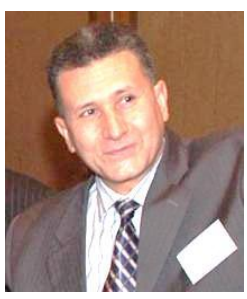

Lakhdar Chouireb was born on May 28, 1957 in Biskra, Algeria. After succeeding his mathematics baccalaureate in 1975, he graduated from the University of Algiers, Algeria, with a state Engineering degree in Mathematics, in 1980 and he got his M.Sc in 1985. He worked in different company before he joined SONELGAZ the only Gas and Power provider of Algeria. Chouireb Lakhdar was promoted in 2009 as an Analysis and prevision director of the SONELGAZ Department of Strategy and prospective. In 2011 the SONLEGAZ Company worked with the LABGED laboratory and Badji Mokhtar University to develop models and software for the Algerian midlong term load forecasting. His research interests are load forecasting, linear and nonlinear regression and statistical approach for load forecasting. 